\title{
Specialist and Generalist Fungal Parasites Induce Distinct Biochemical Changes in the Mandible Muscles of Their Host
}

\author{
Shanshan Zheng ${ }^{1,2,3}$, Raquel Loreto ${ }^{4,5}$, Philip Smith ${ }^{6}$, Andrew Patterson ${ }^{7}$, David Hughes ${ }^{3,8, *}$ \\ and Liande Wang ${ }^{1,2, *}$ \\ 1 Key Laboratory of Biopesticide and Chemical Biology, Ministry of Education, Fuzhou 350002, China; \\ zhengshanshan0428@gmail.com \\ 2 College of Plant Protection College, Fujian Agriculture and Forestry University, Fuzhou 350002, China \\ 3 Department of Entomology, Pennsylvania State University, University Park, PA 16802, USA \\ 4 Center for Infectious Diseases Dynamics, Pennsylvania State University, University Park, PA 16802, USA; \\ raquelgloreto@gmail.com \\ 5 CAPES Foundation, Ministry of Education of Brazil, Brasilia 70040-020, DF, Brazil \\ 6 Metabolomics Core Facility, Huck Institutes of Life Sciences, Pennsylvania State University, University Park, \\ PA 16802, USA; pbs13@psu.edu \\ 7 Center for Molecular Toxicology and Carcinogenesis, Department of Veterinary and Biomedical Sciences, \\ Pennsylvania State University, University Park, PA 16802, USA; adp117@psu.edu \\ 8 Department of Biology, Pennsylvania State University, University Park, PA 16802, USA \\ * Correspondence: dph14@psu.edu (D.P.); liande_wang@126.com (L.W.)
}

Received: 6 August 2019; Accepted: 10 September 2019; Published: 17 September 2019

check for updates

\begin{abstract}
Some parasites have evolved the ability to adaptively manipulate host behavior. One notable example is the fungus Ophiocordyceps unilateralis sensu lato, which has evolved the ability to alter the behavior of ants in ways that enable fungal transmission and lifecycle completion. Because host mandibles are affected by the fungi, we focused on understanding changes in the metabolites of muscles during behavioral modification. We used High-Performance Liquid Chromatography-Mass/Mass (HPLC-MS/MS) to detect the metabolite difference between controls and O. unilateralis-infected ants. There was a significant difference between the global metabolome of $O$. unilateralis-infected ants and healthy ants, while there was no significant difference between the Beauveria bassiana treatment ants group compared to the healthy ants. A total of 31 and 16 of metabolites were putatively identified from comparisons of healthy ants with O. unilateralis-infected ants and comparisons of B. bassiana with O. unilateralis-infected samples, respectively. This result indicates that the concentrations of sugars, purines, ergothioneine, and hypoxanthine were significantly increased in O. unilateralis-infected ants in comparison to healthy ants and B. bassiana-infected ants. This study provides a comprehensive metabolic approach for understanding the interactions, at the level of host muscles, between healthy ants and fungal parasites.
\end{abstract}

Keywords: behavioral manipulation; muscular atrophy; fungal pathogens; mandible muscle

\section{Introduction}

Some parasites have evolved to manipulate the biting behavior of their insect's hosts, causing infected individuals to affix themselves by their mandibles to vegetation [1]. A notable example of this is the behavior changes in ants when infected by species of fungi Ophiocordyceps unilateralis sensu lato (s.l.) [2,3]. Fungi species in this group have evolved to control the biting behavior of ants before death, an adaptive function for the fungus because it removes the infected individual from 
the ant colony where its development is prevented by the cleaning behavior of the resident ant [4]. Once manipulated the fungus kills the ant and uses its body to produce spores that are released to fall to the ground where they infect foraging ants.

Previous morphological studies on this host-parasite system revealed that the muscle fibers of the head experience significant atrophy concomitant with fungal infection $[5,6]$. Moreover, fungal cells penetrated between the muscle fibers [6]. Because fungi are well known to secrete chemicals that affect their environment [7], a parsimonious explanation is that the observed atrophy may be due to secreted chemicals. Recent transcriptomics work supports this hypothesis, highlighting a large and dynamic secretome of the fungus during manipulation. Although transcriptomics is an important tool that provides insights into the control of insect tissues by fungal pathogens, an additional level of detail could be achieved by analyzing the changes in chemicals directly using a metabolomics approach.

Other recent studies of the metabolome of entomopathogenic fungi have been described and have also revealed aspects of host-parasite interactions [8-10]. In fungi, secondary metabolites are secreted through the cell wall and these play an important role in most environmental interactions [7]. A previous ex vivo metabolite study on $O$. unilateralis demonstrated that guanidinobutyric acid and sphingosine are two candidate compounds that are secreted in the presence of host tissues and may be involved in the observed morphological changes that underlie the behavioral manipulation. It was also demonstrated that $O$. unilateralis reacts differently to the brains of different ant species and when grown alongside muscles versus brains, as well as reacting differently to live versus dead tissue [11].

In the present study, we went beyond ex vivo studies to assay the metabolites of O. unilateralis-infected Camponotus castaneus ants at the time of manipulation. We focused on the mandibular muscles of ants since this is the most crucial tissue involved in the biting behavior that typifies this complex behavioral manipulation [6]. Since changes in the metabolome may reflect general pathology we also examined the metabolome of muscles of ants infected by the generalist fungal pathogen Beauveria bassiana as a positive control. The fungus B. bassiana can infect and kill ants (and other insects) but does not control host behavior. We used healthy ants as a negative control. We compared the metabolomes of these three groups using High-Performance Liquid Chromatography-Mass Spectrometry (HPLC-MS). We found that there were significant differences in the muscles of ants according to treatment, and the differences were most pronounced in behaviorally manipulated ants.

\section{Results}

We used High-Performance Liquid Chromatography- Mass Spectrometry (HPLC-MS) to detect the metabolite difference between controls and O. unilateralis-infected ants. We found a significant difference between the global metabolome of $O$. unilateralis-infected ants and healthy ants, while there was no significant difference between the B. bassiana-treatment ants group compared to the healthy ants.

\subsection{Difference between the Metabolome of Fungal-Infected Ant Muscles}

We compared the global metabolome of ant mandible muscles across three treatments (Figure 1). The PCA showed that muscles of ants infected by O. unilateralis were markedly different from the muscles of either the positive controls (ants infected by B. bassiana) or healthy ants (Figure 1A,B). The clustering showed that the metabolites in the muscles from behaviorally manipulated ants (infected by $O$. unilateralis), that were biting at the time of sampling, were clearly different from metabolites in the other two groups. Moreover, the two other muscle categories (infected with the fungus B. bassiana and non-infected) had a less pronounced difference between each other compared to the O. unilateralis-infected ants' mandible muscles. 

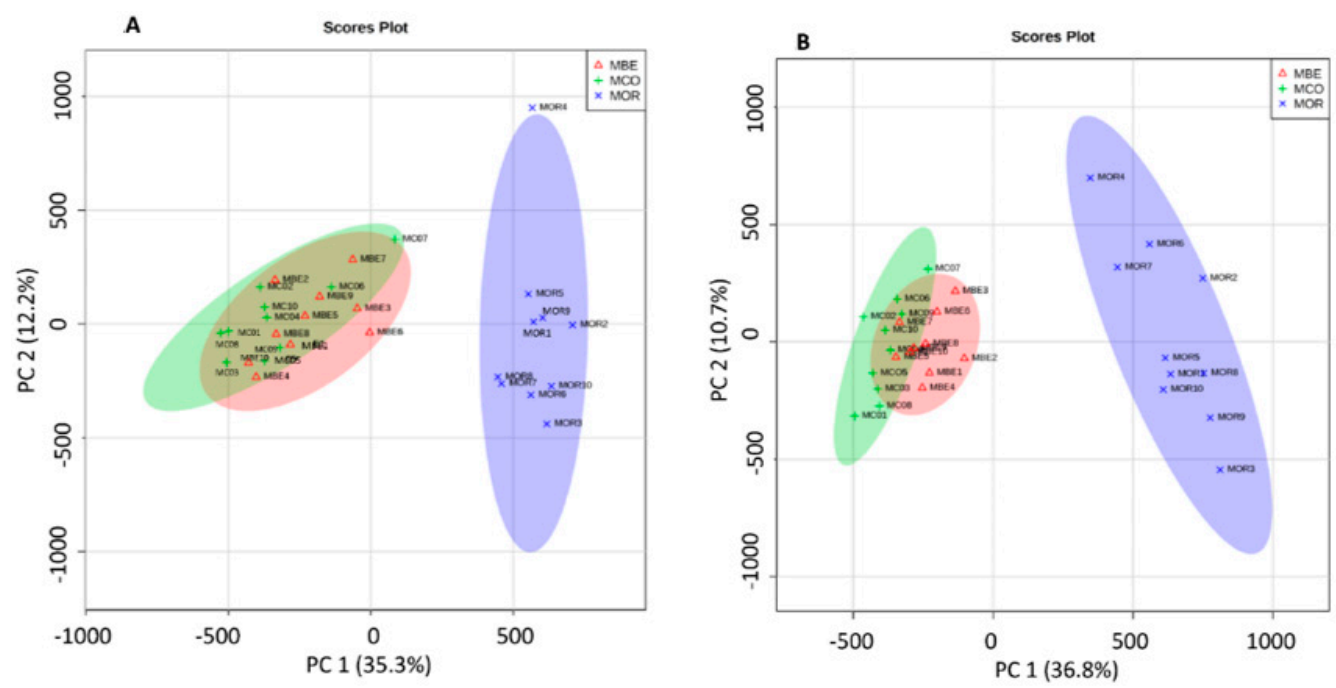

Figure 1. Overview of the three treatments with principal component analysis (PCA). Scores plot of a PCA model from Ophiocordyceps unilateralis-infected (blue, MOR), Beauveria bassiana-infected (red, $\mathrm{MBE}$ ), and healthy ants' (green, MCO) muscle samples. (A) PCA plot showing the clustering of three different treatment samples from positive ion MS/MS feature data. (B) PCA plot showing the clustering of three different treatment samples from negative ion MS/MS feature data.

\subsection{Identifying Tissues Metabolites between Behaviorally Manipulated Ants and Controls}

To systematically delineate metabolite changes associated with the modulation of the biting behavior, we performed metabolic profiling of infected and healthy ants by using HPLC-QTOFMS. OPLS-DA showed a significant difference in muscles between the two treatments. These sample points, with both positive and negative ion data, were divided into two separate groups in the score plot (positive, $\mathrm{R}^{2} \mathrm{Y}=0.997, \mathrm{Q}^{2}=0.919, p<0.001$; negative, $\mathrm{R}^{2} \mathrm{Y}=0.990, \mathrm{Q}^{2}=0.964, p<0.001$ ), separating the healthy and O. unilateralis-infected insects (Figure 2A,B).

To try and identify the metabolites secreted by infected and healthy ants, we used SIMCA-P +13 to perform OPLS-DA and S-plot analysis (Figure 2B,D). We used a $p$ (corr) $> \pm 0.8$ to identify 382 and 247 significantly different retention time/mass to charge ratio signals for the positive and negative metabolites, respectively.Among the metabolites that were identified by MS/MS, 31 chemical compounds were putatively identified by using Metlin Database. They included sugars, fatty acids, nucleosides, amino acids, and antioxidant chemicals. Overall, several metabolites displayed considerable differences between O. unilateralis and healthy ants (Table 1). The MS/MS was matched with the 23 commercial standards.

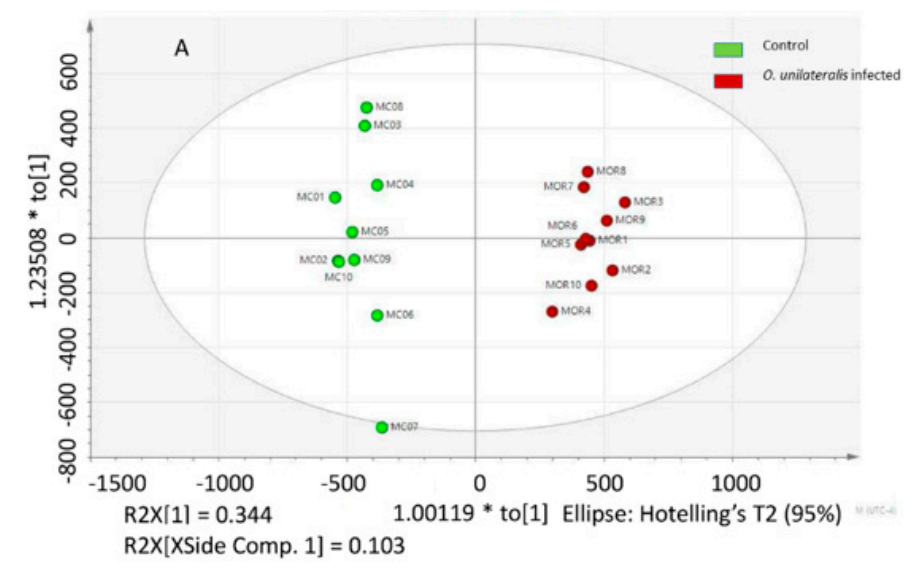

Figure 2. Cont. 

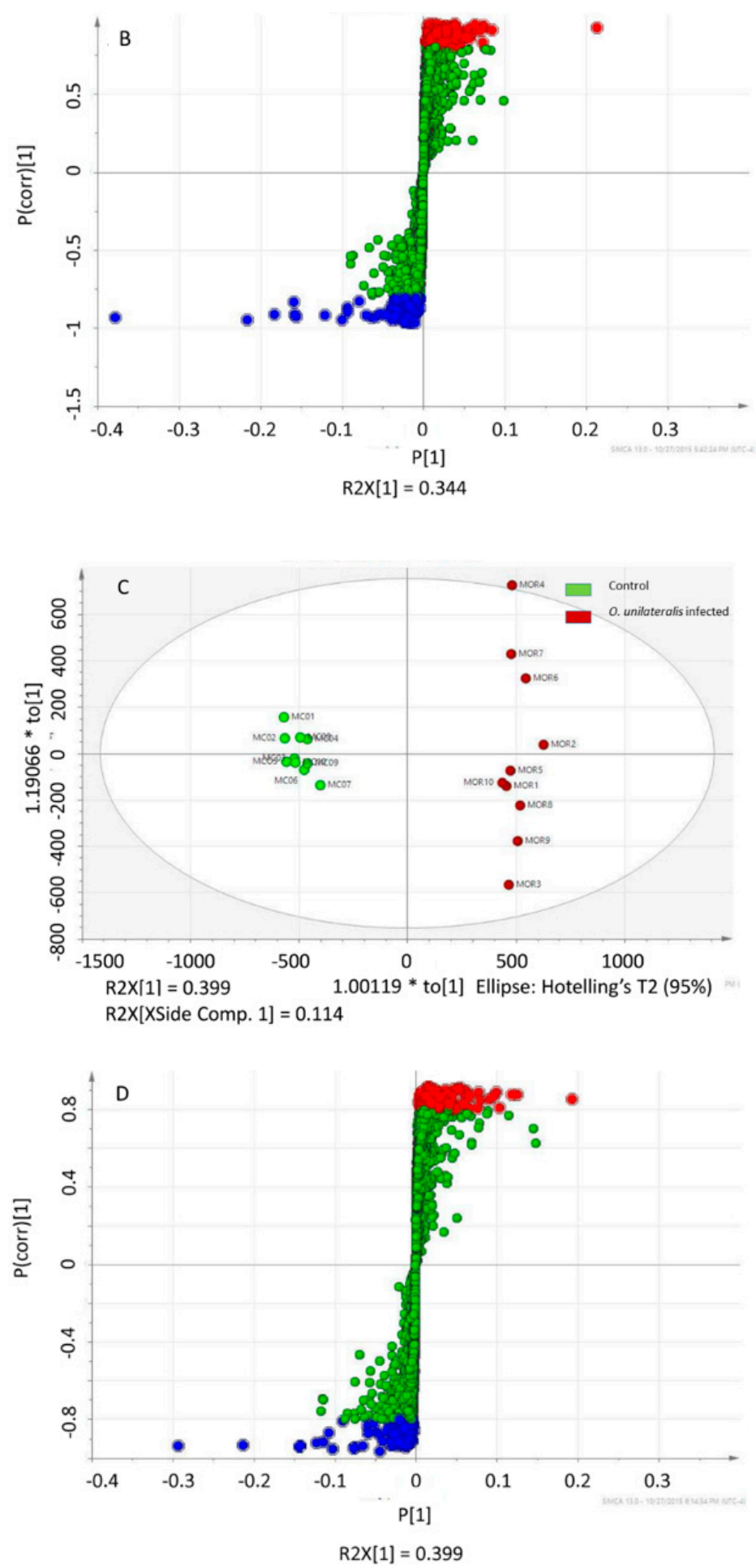

Figure 2. Differential metabolic profiles in muscle between healthy and O. unilateralis-infected ants. (A) Orthogonal projection for latent structures-discriminant analysis (OPLS-DA) score plot separating healthy ants (green) and O. unilateralis-infected (red) samples from positive ion feature data. (B) S-plot analysis of all $O$. unilateralis-infected samples against all healthy ants' samples from positive ion feature data. (C) OPLS-DA score plot separating healthy ants (green) and O. unilateralis-infected (red) samples from negative ion feature data. (D) S-plot analysis of all O. unilateralis-infected samples against all healthy ants' samples from negative ion feature data. 
Table 1. Compounds identified by HPLC-MS/MS analysis from healthy ants compared with Ophiocordyceps unilateralis-infected ants.

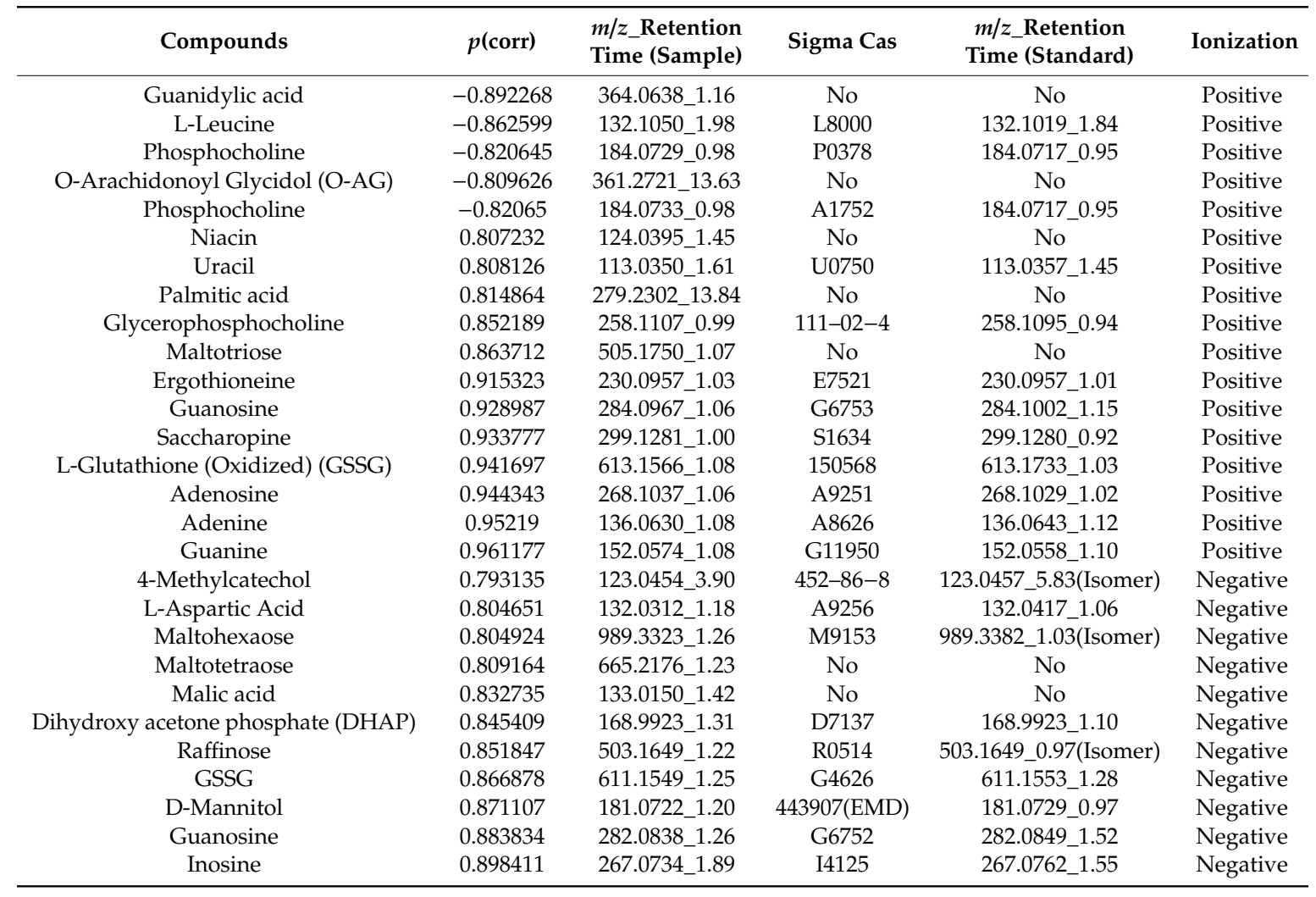

\subsection{Different Metabolites in the Comparison between Ophiocordyceps unilateralis s.l. and Beauveria} bassiana-Infected Ants

HPLC-MS/MS was performed to determine the relative levels of metabolites in mandiblemuscle infected by $O$. unilateralis ants that caused the characteristic biting behavior and muscle infected by $B$. bassiana fungi that did not. Muscle samples were clustered into two distinct groups in the OPLS-DA score plot (positive, $\mathrm{R}^{2} \mathrm{Y}=0.980, \mathrm{Q}^{2}=0.905, p<0.001$; negative, $\mathrm{R}^{2} \mathrm{Y}=0.985, \mathrm{Q}^{2}=0.935$, $p<0.001$ ) (Figure 3A,C). The results showed that the muscles of behaviorally manipulated ants had 455 metabolites (277 positive and 184 negative) significantly up- or down-regulated compared to the muscles of ants infected by the generalist fungus, B. bassiana (Figure 3B,D). The 16 putatively identified metabolites are shown in Table 2. Using commercial standards we could confirm 11 metabolites.

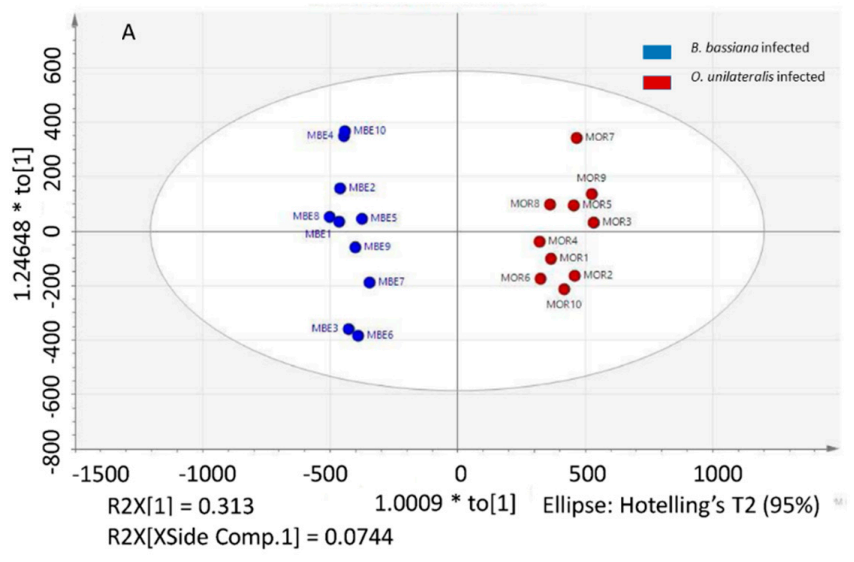

Figure 3. Cont. 

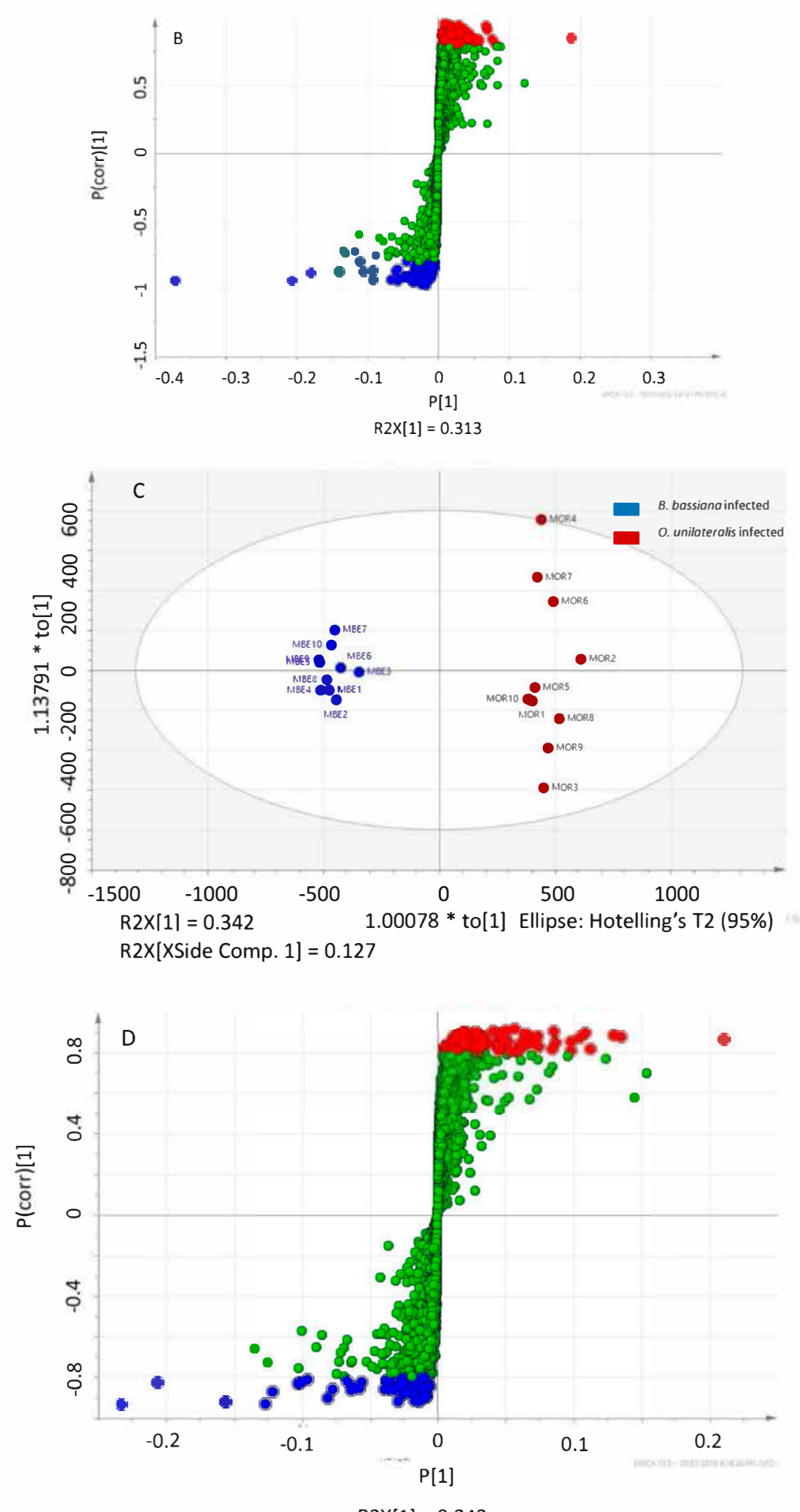

$R 2 X[1]=0.342$

Figure 3. Differential metabolic profiles in muscle between Beauveria bassiana- and Ophiocordyceps unilateralis-infected ants. (A) OPLS-DA score plot separating B. bassiana-infected (blue) and O. unilateralisinfected (red) samples from positive ion feature data. (B) S-plot analysis of all B. bassiana-infected samples against $O$. unilateralis ants' samples from positive ion feature data. (C) OPLS-DA score plot separating B. bassiana-infected (blue) and O. unilateralis-infected (red) samples from negative ion feature data. (D) S-plot analysis of all B. bassiana-infected samples against O. unilateralis-infected ants' samples from negative ion feature data. 
Table 2. Compounds identified by HPLC-MS/MS analysis from Beauveria bassiana-infected ants compared with Ophiocordyceps unilateralis-infected ants.

\begin{tabular}{|c|c|c|c|c|c|}
\hline Compounds & $p$ (corr) & $\begin{array}{l}m / z \_ \text {Retention } \\
\text { Time (Sample) }\end{array}$ & Sigma Cas & $\begin{array}{l}m / z_{\text {R Retention }} \\
\text { Time (Standard) }\end{array}$ & Ionization \\
\hline Guanine & -0.915064 & 152.0570_1.09 & G11950 & 152.0560_1.04 & Positive \\
\hline Ergothioneine & -0.908576 & 230.0964_1.01 & E7521 & 230.0957_1.01 & Positive \\
\hline Guanosine & -0.88987 & 284.0967_1.06 & G6753 & 284.1002_1.15 & Positive \\
\hline Hypoxanthine & -0.885579 & 137.0459_1.06 & H9377 & 137.0461_1.03 & Positive \\
\hline Adenosine & -0.850788 & 268.1037_1.06 & 116833 & 268.1029_1.02 & Positive \\
\hline Adenine & -0.842341 & 136.0621_1.06 & A8626 & 136.0643_1.12 & Positive \\
\hline 4-Hydroxycinnamoylmethane(4-HM) & -0.805273 & 163.0735_9.68 & No & No & Positive \\
\hline Glycemphosphocholine & -0.803298 & 258.1095_0.99 & & 258.1095_0.94 & Positive \\
\hline AMP & 0.800485 & 348.0707_1.08 & A1752 & 348.0688_1.03 & Positive \\
\hline Guanidylic acid & 0.883806 & 364.0638_1.16 & No & No & Positive \\
\hline Maltopentaose & 0.806347 & 827.2799_1.16 & No & No & Negative \\
\hline Maltoteraose & 0.814304 & 665.2176_1.23 & No & No & Negative \\
\hline Maltoheptaose & 0.837664 & 1151.3911_1.16 & no & No & Negative \\
\hline Raffinose & 0.851338 & 503.1664_1.22 & R0514 & 503.1664_0.97(Isomer) & Negative \\
\hline D-Mannitol & 0.855712 & 181.0722_1.20 & 443907(EMD) & 181.0729_0.97 & Negative \\
\hline Inosine & 0.904759 & 267.0734_1.89 & I 4125 & 267.0762_1.55 & Negative \\
\hline
\end{tabular}

From S-plot analysis, we found 569 features were significantly increased or decreased in the mandible muscles of $O$. unilateralis-infected ants compared to healthy ants. We found 380 features were significantly increased or decreased in O. unilateralis-infected ants compared to B. bassiana-infected ants. Only 202 of the increased and 96 decreased ion features were shared when mandible muscles of O. unilateralis-infected ants were compared to mandible muscle B. bassiana-infected ants (Figure 4). To visualize the significantly changed mandible muscle metabolites we created a heat map using the Euclidean distance measure (Figure 5).

The identified metabolites were imported into the MetaboAnalyst online database for metabolic pathway enrichment analysis. The hypergeometric distribution method was used for analysis to calculate the $p$-value of each metabolic pathway. Enrichment analysis and topological analysis of the pathways in which the differential metabolites are located revealed a pathway with a high correlation with metabolites ( $p$-value less than 0.05 , influence value greater than 0.1 ) (Figure 6 ). The most relevant metabolic pathway is the purine metabolic pathway $\left(p=1.3968 \times 10^{-4}\right.$, impact $\left.=0.14393\right)$.
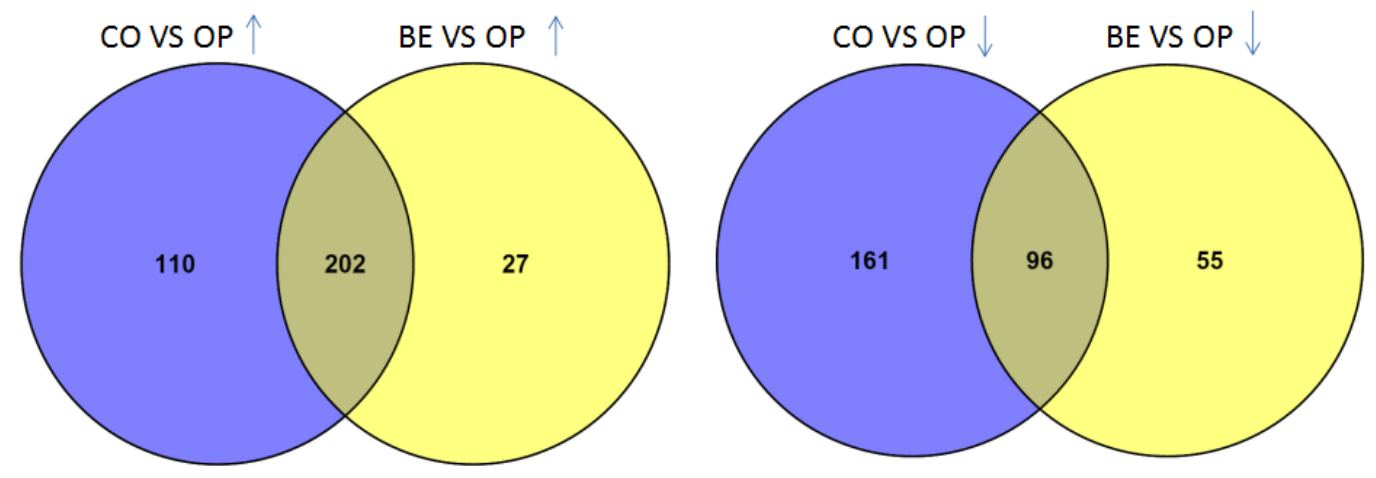

Figure 4. Venn diagram representing overlap between the two groups samples comparing all ion features found to be increased $(\uparrow)$ and decreased $(\downarrow)$ in O. unilateralis-infected mandible muscle samples based on $p$ (corr) $>0.8$ and $p$ (corr) $<-0.8$, respectively (CO: control; OP: O. unilateralis-infected ants; BE: B. bassiana-infected ants). 


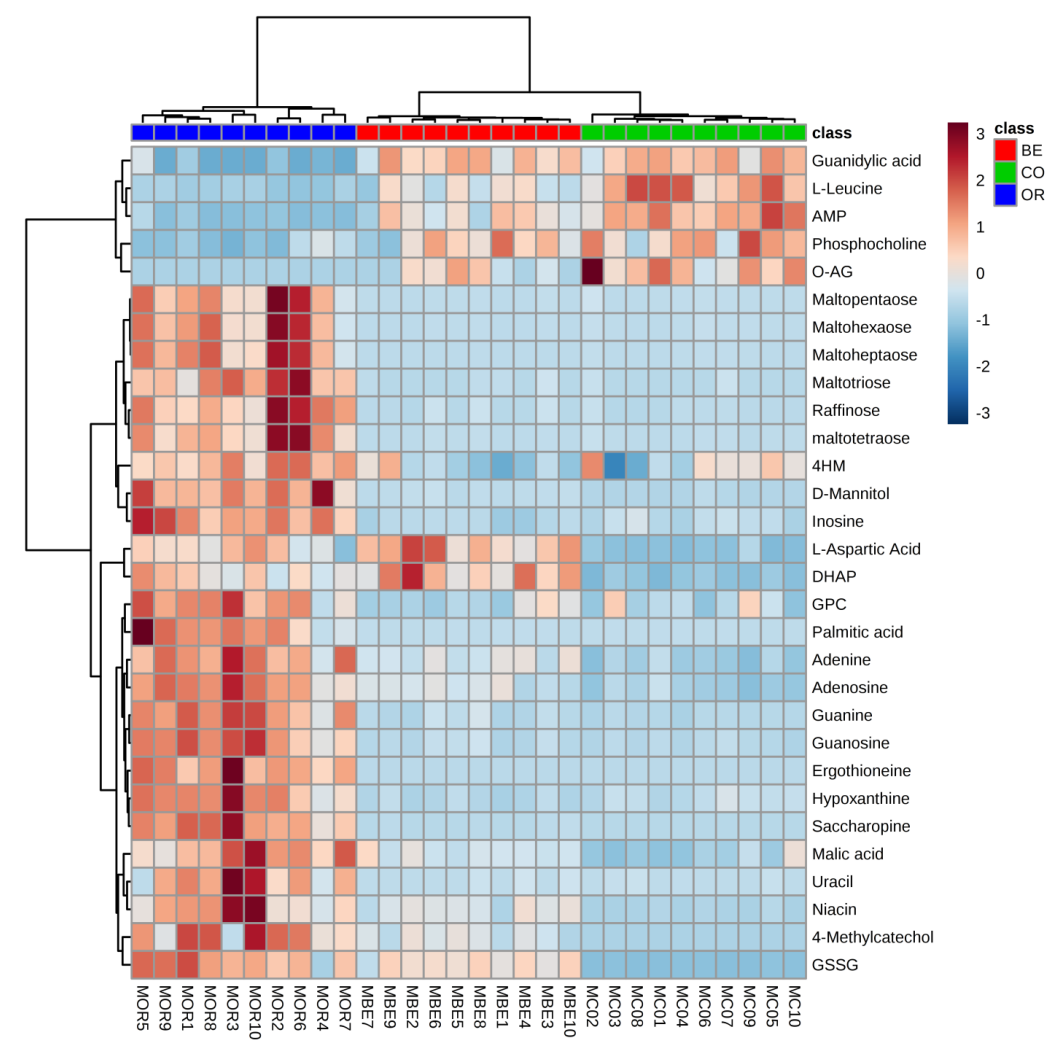

Figure 5. Heat maps of 30 differently identified metabolites from the mandible muscles of the three different treatment group ants: healthy ants (CO, colored green); B. bassiana-infected ants (BE, colored red); and O. unilateralis-infected ants (OR, colored blue). Each metabolite was represented by a single row of colored boxes, where columns represented different samples. Metabolite levels are scaled by colors. The red labels show high intensities levels and light blue labels mean low intensities of metabolites levels using Euclidean distance measure.

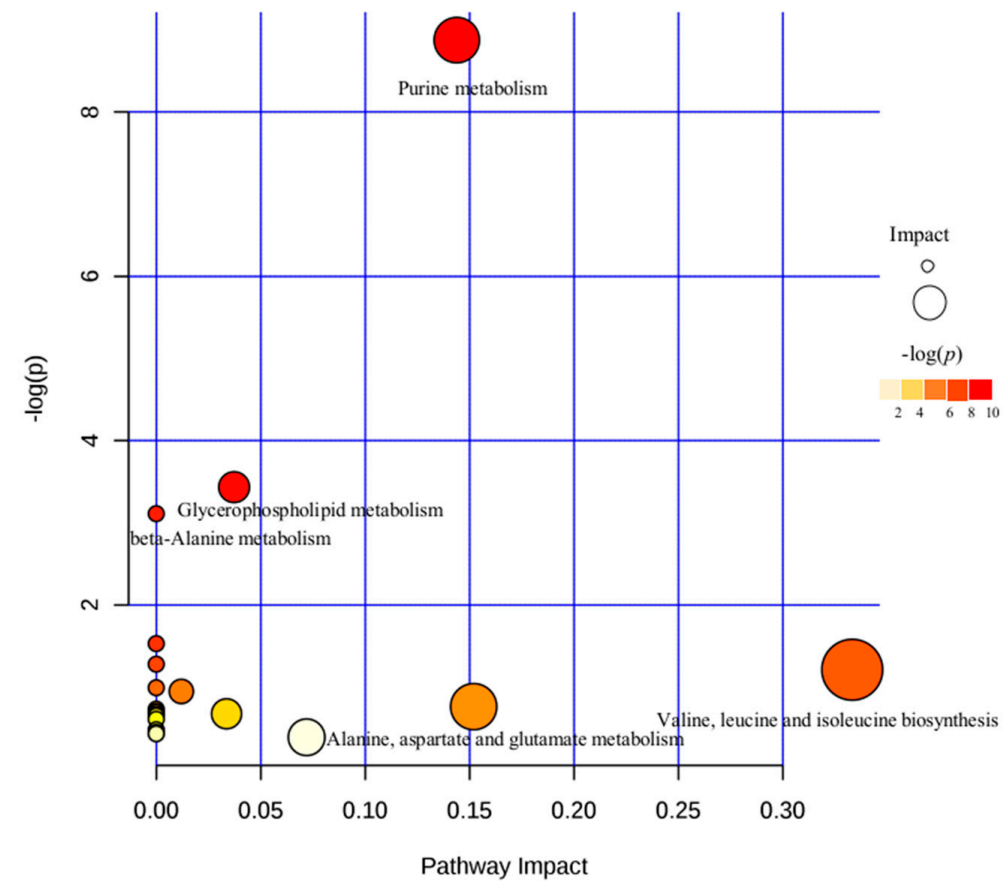

Figure 6. Pathway analysis for the O. unilateralis group vs. B. bassiana and the uninfected group. 
When the metabolites from O. unilateralis-infected ants vs. B. bassiana-infected and non-infected samples were compared, we observed a notable increase of purines in terms of the levels of adenine, guanine, uracil, and hypoxanthine. Additionally, levels of adenosine, composed of adenine and ribose sugar, were significantly higher in behaviorally manipulated ants. On the other hand, adenosine monophosphate (AMP) levels were lower in O. unilateralis-infected ants compared with B. bassiana-infected samples. Sugar levels were higher in O. unilateralis-infected ants, with higher levels of maltohexaose, raffinose, and maltoheptaose. We identified erogothione in O. unilateralis-infected ants. Our results show that levels of erogothione, purines, and sugar were significantly increased after infection, indicating they could have potential role in regulating biting behavior of the ants.

\section{Materials and Methods}

\subsection{Camponotus Castaneus Ant-Rearing and Treatments}

Insects used for the experiments were selected from three C. castaneus colonies (KFM1, KFM22, and KFMX), which have approximately 200 workers, obtained in South Carolina, USA in August 2014. Ants were reared in three large plastic boxes $(27 \mathrm{~cm} \times 19 \mathrm{~cm} \times 10 \mathrm{~cm})$ in the laboratory and supplied with 30\% sugar-water solution and water. Colonies were maintained under a standard $12 \mathrm{~h}$ light, $12 \mathrm{~h}$ dark photoperiod cycle in $24 \pm 1{ }^{\circ} \mathrm{C}$ laboratory conditions over the course of one week before the experiment. Samples were collected from there colonies. A total of 145 ants were used in the experiments described here.

\subsection{Ophiocordyceps unilateralis s.l. Infection}

The pathogen O. unilateralis was obtained from infected C. castaneus ants in South Carolina, USA. The fungus $O$. unilateralis was grown on potato dextrose agar (PDA) medium in petri dishes. The injection of ants with the fungus was done following the protocol described by de Bekker et al. [11]. Briefly, part of the fungal colony, one-eighth of the fungal culture, which had grown to an approximately 1-cm diameter, was collected, suspended in $500 \mu \mathrm{L}$ Grace's medium solution, and placed in a tissue lyser for $30 \mathrm{~s}$ to mechanically break the colony up into small hyphal fragments. The solution of hyphal fragments was then diluted four times before use. For each colony, 30 ants were infected with O. unilateralis. Each ant was injected with $1 \mu \mathrm{L}$ of fungal suspension through the intersegmental membranes underneath the forelegs. In total, 90 ants were used for the O. unilateralis infection. Once infected we returned infected ants to their colony and observed them for between 15 and 25 days post infection and only sampled ants that were behaviorally manipulated to bite.

\subsection{Beauveria Bassiana Infection}

We used the generalist fungal pathogen B. bassiana as a positive control. It is capable of infecting ants (and many different orders of insects, besides Hymepnotera) but does not adaptively manipulate ant behavior. Conidia of B. bassiana were obtained from Dr. Nina Jenkins, Pennsylvania State University. The concentration of $B$. bassiana conidia solution used for the experiment was $5 \times 10^{9}$ conidia per $\mathrm{mL}$ in $0.05 \%$ Tween 80 . For B. bassiana infection, $3 \mu \mathrm{L}$ of conidia solution were spread over the intersegmental tissue that lies under the first pair of legs, where they attach to the thorax. We used this surface infection method rather than injection because ants injected with B. bassiana normally die within $3-5$ days because the growth of this fungus is more rapid than O. unilateralis. Since injection itself can occasionally lead to the death of ants, it is not always possible to determine whether injected ants died from fungal infection or from the injection procedure. For each colony, 15 ants were used for B. bassiana infection. Then they were moved to the large plastic boxes, fed, and placed under the same laboratory conditions as O. unilateralis-infected ants. Ants infected with B. bassiana are not behaviorally manipulated prior to death. To establish that the ants were dying we checked their behavior every $15 \mathrm{~min}$ from the time the first dead ants appeared. It was clear when ants were moribund and close to death because they would have pronounced difficulties in walking and their body would be curled 
over. We are therefore confident that we collected ants which were close to death. Collected ants were snap frozen using liquid nitrogen, before being stored at $-80^{\circ} \mathrm{C}$.

\subsection{Healthy Control Ants}

For each colony, 10 ants were randomly selected and moved to a new small plastic cage and fed under the same conditions as other two treatments for 15 days before they were snap frozen with liquid nitrogen and collected.

\subsection{Metabolite Assay}

All nine cages ( 3 colonies $\times 3$ treatments) were placed under a 13-h light, 11-h dark photoperiod cycle and humidity was maintained at or above $60 \%$ relative humidity with temperature at $28{ }^{\circ} \mathrm{C}$ constantly (SI Appendix, Figure S1). We used 30 ants for the metabolite study: 10 O. unilateralis-infected ants, 10 B. bassiana-infected ants, and 10 healthy ants (SI Appendix, Table S1). Under a dissecting microscope, the cuticle of the head was opened, on ice, to reveal ant muscles. All the extra tissues were removed to expose the muscles. The muscles of the right side of the head were transferred into a 2-mL graduated cryogenic tube and stored at $-80{ }^{\circ} \mathrm{C}$ until analysis. Only one person performed all the dissections in order to minimize variability in dissection methods. The sample extraction, metabolite separation, and detection followed the methodology described previously by de Bekker et al. [11]. Briefly, the extraction solvent system was 50/50 (v/v) methanol/water with $1 \mu \mathrm{mL}$ of chlorpropamide as an internal standard. To collect the metabolites, we added $105 \mu \mathrm{L}$ extraction solvent to each sample and placed this in a Precellys homogenizer (Bertin Technologies, Rockville, MD, USA) for two cycles at $6800 \mathrm{rpm}$ for $30 \mathrm{~s}$. After homogenization and centrifugation at 20,000 $\times g\left(4^{\circ} \mathrm{C}\right)$ for $15 \mathrm{~min}, 75 \mu \mathrm{L}$ of the supernatant were transferred into a low-volume plastic auto sampler vial. Samples $(5 \mu \mathrm{L})$ were separated by reverse phase HPLC using a Prominence 20 UFLCXR system (Shimadzu, Columbia, MD, USA) with a Waters (Milford, MA, USA) BEH C18 column (100 mm $\times 2.1 \mathrm{~mm}, 1.7$-um particle size) and maintained at $55{ }^{\circ} \mathrm{C}$ with a 20 -min aqueous acetonitrile gradient at a flow rate of $250 \mu \mathrm{L} / \mathrm{min}$. Solvent A was HPLC-grade water with $0.1 \%$ formic acid and Solvent B was HPLC grade acetonitrile with $0.1 \%$ formic acid. The initial conditions were $97 \% \mathrm{~A}$ and $3 \% \mathrm{~B}$, increasing to $45 \% \mathrm{~B}$ at $10 \mathrm{~min}$, and $75 \% \mathrm{~B}$ at $12 \mathrm{~min}$ and holding at $75 \% \mathrm{~B}$ to $17.5 \mathrm{~min}$ before returning to the initial conditions. The eluate was delivered into a 5600 (QTOF) triple TOF using a Duospray ${ }^{\mathrm{TM}}$ ion source (all AB Sciex, Framingham, MA, USA). The capillary voltage was set at $5.5 \mathrm{kV}$ in positive ion mode and $4.5 \mathrm{kV}$ in negative ion mode, with a decluttering potential of $80 \mathrm{~V}$. The mass spectrometer was operated in Information-Dependent Acquisition (IDA) mode with a 100-ms survey scan from 100 to $1200 \mathrm{~m} / \mathrm{z}$ for up to $20 \mathrm{MS} / \mathrm{MS}$ product ion scans $(100 \mathrm{~ms})$ per duty cycle using collision energy of $50 \mathrm{~V}$ with a $20-\mathrm{V}$ spread. The running order of the samples was randomized to minimize the effect of any long-term changes in instrument performance.

\subsection{Statistics Analysis}

Normalization is a method which accounts for different dilutions of samples by scaling the spectra to the same virtual overall concentration [12]. Normalization using the total spectral intensity can be used for MS-based metabolomics data sets, and this reduces the effect of a single prominent component in the chromatogram such as xenobiotics or artifacts, which would not be appropriate measures of concentration [13]. Chromatograms were aligned, and features (retention time $\mathrm{m} / \mathrm{z}$ pairs) were extracted from the LC-MS data using MarkerView and normalized by using the total area sums. The features were used for metabolomics profiling by principal component analysis (PCA) [14] and heat maps with MetaboAnalyst 3.0 online (http://www.metaboanalyst.ca/); orthogonal projection for latent structures-discriminant analysis (OPLS-DA) [15] and analysis of variance (ANOVA) were carried out with SIMCA-P+13 (Umetrics). The OPLS-DA modelled covariance (cov $\left(t_{p}, X\right)$, also known as $p$ (corr)), was plotted against the chemical shift and the plot was colored accordingly.The $\mathrm{R}^{2} \mathrm{Y}$ and $\mathrm{Q}^{2}$ are the two parameters that judge the model. All data were autoscaled (mean-centered and scaled to 
unit variance). CV-ANOVA was used for the significance testing of the OPLS-DA model, including sum of squares (SS), degrees of freedom (DF), mean square (M), $p$-value ( $p$ ), and standard division (SD). Scores in metabolite changes to fungi infection were compared between groups using the orthogonal partial least squares discriminant analysis (OPLS-DA) score.

\subsection{Metabolite Identification}

Metabolites were identified by a comparison of their product ion mass spectra to those present in the online Metlin Database (https://metlin.scripps.edu/metabo_search_alt2.php). Retention times and peak areas of the metabolites of interest were extracted and automatically integrated with Peak View (AB Sciex), which was also used for quantitative review of HPLC-MS/MS data in the identification of metabolites. Each putatively identified metabolite was verified with standards if they were commercially available. Metabolite standards were prepared at a fine concentration of $10 \mathrm{uM}$ in $50 / 50(v / v)$ methanol/water and prepared fresh before use.

\section{Discussion}

In order to extend our knowledge about the biochemical changes that occur inside the muscles of insects affected by fungal pathogens, we used metabolomics to compare some fractions of the biochemical composition of muscles tissue of carpenter ants (C. castaneus) infected by O. unilateralis, which is known to induce a stereotypical biting behavior prior to host death. Using HPLC-based metabolomics analysis we discovered candidate metabolites for the O. unilateralis-infected ants and those infected by the generalist fungal pathogen, B. bassiana, which does not affect behavior, but can infect and kill ants. For O. unilateralis-infected ants, 31 metabolites were identified and confirmed (using their commercially available standards), including ergothioneine, hypoxanthine, and saccharopine. In addition, 14 metabolites, including niacin, glycerophosphocholine, and maltotriose (isomer), were putatively identified (without standard).

When comparing healthy ants and B. bassiana-infected samples to O. unilateralis-infected ants, we observed a significant decrease in L-leucine of O. unilateralis-infected ants. Leucine is an essential amino acid and we were able to confirm its presence with the use of a commercial standard. Animals cannot synthesize leucine and bioaccumulation can occur in animal tissues, as a result of consuming organisms which produce it. Several studies have suggested that leucine is a nutrient regulator of muscle protein synthesis by activating components of the mammalian target of rapamycin signaling cascade [16-20]. In mammals, cancer can result in cachexia, which is a reduction in muscle mass associated with tumor growth, and leucine supplementation was shown to decrease this in rats [21]. In ants infected by the behaviorally manipulating fungus $O$. unilateralis, previous work has shown a significant decrease in mitochondria which are the power generators of the cells and the source of ATP and sarcoplasmic reticula that provide calcium for actin-myosin binding [6]. We suggest that the behaviorally manipulating fungus $O$. unilateralis may be targeting muscle cells by reducing their available energy and $\mathrm{Ca}^{2+}$ and depleting leucine levels to prevent muscle regeneration.

An intriguing feature of this study was the concentration of hypoxanthine, which was significantly increased in the mandible muscles of $O$. unilateralis-infected ants compared to either healthy ants or the positive control of B. bassiana-infected ants. For survival and growth, certain pathogens require hypoxanthine [22-24] and obtain this necessary nutrition by salvaging purine from their hosts. In addition, hypoxanthine-guanine phosphoribosyl transferase, a known protease, is critical to this process [25]. Studies have reported that hypoxanthine is a catabolic product of ATP [26,27]. On the other hand, the breakdown of ATP also produces AMP and is associated with energy use in cellular processes. The identified hypoxanthine is consistent with this catabolic cycle and it was observed that AMP content decreased in metabolite abundance for O. unilateralis-infected ants. Based on these results, we suggest that the infection by O. unilateralis might therefore stimulate ATP breakdown, which is line with previous observations on a significant reduction in mitochondria in the muscles of infected ants [6]. This mechanism is likely due to an enhancement in glucose consumption by the 
fungus growth, which also results in an increased rate of hypoxanthine production. Additionally, Feet et al. [28] and Phillis et al. [29] reported that high concentrations of extracellular hypoxanthine induced damage to neural tissue of the cerebral cortex. We know from histological work that a distinct atrophy occurs with widely spaced myofibrils and a previous suggestion was that muscle innervation was impacted [6]. In this study the increased concentration of hypoxanthine may suggest a mechanism by which the fungus affects the motor neurons of the muscles it is targeting.

Our result also suggests that saccharopine is strongly increased in mandible muscles after O. unilateralis infection. Saccharopine is a precursor of lysine, which is an essential amino acid. Some studies suggest that in fungi, lysine is synthesized through the saccharopine pathway, whereas in plants, bacterium, and animals, the pathway functions in lysine degradation [30-34]. Lysine biosynthesis is essential for within host entomopathogenic fungal growth [35]. Specifically, saccharopine occurs as an intermediate of lysine metabolism in the alpha-aminoadipate pathway of fungi [36-39], and can be isolated from the fungal fruiting body [40]. Therefore, the observation of saccharopine accumulated in mandible muscle of the ant suggests that this metabolite is produced by fungi using the host resources. However, the host could be a source. Outside of the central nervous systems lysine is synthesized by the aminoadipate pathway. Inside the brain lysine is synthesized by the pipecolate pathway [41]. The pipecolate pathway is mediated by lysine-alpha-ketoglutarate reductase and converts L-lysine to saccharopine [42]. This suggests that the cause for increased abundance of saccharopine observed in our system may be more complex, as both the host and the parasite are putative sources. This, interestingly, also provides a window for manipulation of the host. Because neither the controls nor the B. bassiana-infected samples showed the presence of saccharopine it is possible that the parasite stimulates the host to produce this compound. This probable influence could be exacted via the direct manipulation of the host, the parasite-specific immunological response of the host, or otherwise. Irrespective of the source (parasite vs. host), we suggest that saccharopine may play an important role in this host-fungal pathogen interaction. More research is needed to confirm the causal mechanism of increased saccharopine abundance.

It was also notable that the relative sugar level was notably increased in the mandible muscles of O. unilateralis-infected ants. Sugars play important roles in non-self-recognition, and distinct sugar signatures may elicit different immune responses [43]. In the plant-fungal antagonistic interactions we know fungi can rapidly metabolize soluble sugars of the host (glucose, fructose, and sucrose) and convert them into their own metabolites [44]. On the other hand, sugar can also promote formation of fungal tissue inside the host $[45,46]$. We know that $O$. unilateralis uses its host cadaver as a carbon source and sequesters host metabolic stores for its own continuous spore production. This results in fungal tissue differentiation in the post-mortem ant where a carbon-nitrogen rations analysis showed by energy storage and transport [5]. In this study, the increased level of sugars also suggests that O. unilateralis likely produces enzymes that have sugar-related activity.

When comparing healthy ants and B. bassiana-infected samples to O. unilateralis-infected ants, we observed a significant increase in ergothioneine of $O$. unilateralis-infected ants. Ergothioneine is a naturally occurring betaine of histidine, wshich was found at high levels in our samples. It is only synthesized by bacteria and fungi, and accumulates in human and animals tissues in cells [47-50]. A great number of studies have suggested that ergothioneine can influence a variety of animal behaviors (including the regulation of the biosynthesis precursor hercynine [51,52]), inhibit oxidative damage in cells $[53,54]$, and scavenge free radicals $[55,56]$. However, the underlying scavenging reaction of ergothioneine differs from reduced glutathione [57]. Given the role of antioxidants, ergothioneine is believed to protect conidia in the life cycle between condiogensis and germination from the toxicity of peroxide. Bello et al. [58] postulated that ergothioneine has a greater impact on conidial survival and germination than on hyphal growth. We know from previous studies that at the time of manipulation the population of the fungal colony is very high, likely as much as $50 \%$ of the total tissue inside the ant $[2,6]$. The fungus is principally in its hyphal body stage, presenting yeast-like cells rather than hyphae. One possibility is that ergothioneine is important during what is a period of rapid cell 
division by the fungus. A clear understanding of the role of ergothioneine in microbes will advance our knowledge of how this thione enhances microbial virulence and resistance to the host's defense mechanisms to avoid complete eradication [50].

\section{Conclusions}

In summary, this study on the metabolomics of ant muscles when infected by a specialized fungal pathogen that has evolved the ability to manipulate ant behavior highlights key biochemical pathways that are likely important for both manipulation and host exploitation. Information obtained from the metabolite study could lead to a better understanding of how $O$. unilateralis manipulates ant behavior and muscle atrophy. In this study, the relative levels of sugars, purines, and ergothioneine were significantly increased in the O. unilateralis-infected ants compared to B. bassiana-infected ants and control ants. Most of the identified metabolites may play essential roles in manipulation. However, a challenge for metabolomics is the identification and quantification of a large fraction of unknown metabolites in complex biological samples when standards are unavailable. The identified metabolites in this study cannot provide the final answer as to whether a particular metabolite causes this death grip or not.

Supplementary Materials: Supplementary materials can be found at http://www.mdpi.com/1422-0067/20/18/ 4589/s1.

Author Contributions: Data curation, S.Z.; Formal analysis, S.Z.; Funding acquisition, D.H.; Investigation, S.Z.; Methodology, R.L. and P.S.; Resources, S.Z. and R.L.; Software, P.S. and A.P.; Supervision, L.W. and D.H.; Visualization, S.Z.; Writing—original draft, S.Z.; Writing—review and editing, L.W. and D.H.

Funding: This work was supported by China Scholarship Council and also funded by Penn State to David Hughes. RGL was funded by CAPES-Brazil (grant 6203-10-8) and the Pennsylvania State University.

Acknowledgments: We thank Philip Smith for HPLC-MS/MS.

Conflicts of Interest: The authors declare no conflict of interest.

\section{References}

1. Moore, J. Parasites and the behavior of animals. Zool. Afr. 2002, 37, 265.

2. Bekker, C.D.; Ohm, R.A.; Loreto, R.G.; Sebastian, A.; Albert, I.; Merrow, M.; Brachmann, A.; Hughes, D.P. Gene expression during zombie ant biting behavior reflects the complexity underlying fungal parasitic behavioral manipulation. BMC Genom. 2015, 16, 620. [CrossRef] [PubMed]

3. Hughes, D.P.; Araújo, J.P.; Loreto, R.G.; Quevillon, L.; De, B.C.; Evans, H.C. From so simple a beginning: The evolution of behavioral manipulation by fungi. Adv. Genet. 2016, 94, 437. [PubMed]

4. Loreto, R.G.; Elliot, S.L.; Freitas, M.L.; Pereira, T.M.; Hughes, D.P. Long-term disease dynamics for a specialized parasite of ant societies: A field study. PLoS ONE 2014, 9, e103516. [CrossRef] [PubMed]

5. Andersen, S.B.; Gerritsma, S.; Yusah, K.M.; Mayntz, D.; Hyweljones, N.L.; Billen, J.; Boomsma, J.J.; Hughes, D.P. The life of a dead ant: The expression of an adaptive extended phenotype. Am. Nat. 2009, 174, 424-433. [CrossRef]

6. Hughes, D.P.; Andersen, S.B.; Hywel-Jones, N.L.; Himaman, W.; Billen, J.; Boomsma, J.J. Behavioral mechanisms and morphological symptoms of zombie ants dying from fungal infection. BMC Ecol. 2011, 11, 13. [CrossRef]

7. Molnár, I.; Gibson, D.M.; Krasnoff, S.B. Secondary metabolites from entomopathogenic hypocrealean fungi. Cheminform 2010, 27, 1241-1275. [CrossRef]

8. Kloehn, J.; Blume, M.; Cobbold, S.A.; Saunders, E.C.; Dagley, M.J.; Mcconville, M.J. Using metabolomics to dissect host-parasite interactions. Curr. Opin. Microbiol. 2016, 32, 59-65. [CrossRef]

9. Wrońska, A.K.; Boguś, M.I. Harman and norharman, metabolites of the entomopathogenic fungus Conidiobolus coronatus (Entomophthorales), affect the serotonin levels and phagocytic activity of hemocytes, insect immunocompetent cells, in Galleria mellonella (Lepidoptera). Cell Biosci. 2019, 9, 29. [CrossRef] 
10. Boyce, G.R.; Gluck-Thaler, E.; Slot, J.C.; Stajich, J.E.; Davis, W.J.; James, T.Y.; Cooley, J.R.; Panaccione, D.G.; Eilenberg, J.; De Fine Licht, H.H.; et al. Psychoactive plant- and mushroom-associated alkaloids from two behavior modifying cicada pathogens. Fungal Ecol. 2019, 41, 147-164. [CrossRef]

11. Bekker, C.D.; Quevillon, L.E.; Smith, P.B.; Fleming, K.R.; Ghosh, D.; Patterson, A.D.; Hughes, D.P. Species-specific ant brain manipulation by a specialized fungal parasite. BMC Evol. Biol. 2014, 14, 166. [CrossRef] [PubMed]

12. Frank, D.; Alfred, R.; Götz, S.; Hans, S. Probabilistic quotient normalization as robust method to account for dilution of complex biological mixtures. Application in $1 \mathrm{H}$ NMR metabonomics. Anal. Chem. 2006, 78, 4281-4290.

13. Warrack, B.M.; Hnatyshyn, S.; Ott, K.H.; Reily, M.D.; Sanders, M.; Zhang, H.; Drexler, D.M. Normalization strategies for metabonomic analysis of urine samples. J. Chromatogr. B 2009, 877, 547-552. [CrossRef] [PubMed]

14. Wold, S.; Esbensen, K.; Geladi, P. Principal component analysis. Chemom. Intell. Lab. Syst. 1987, 2, 37-52. [CrossRef]

15. Trygg, J.; Wold, S. Orthogonal projections to latent structures (O-PLS). J. Chemom. 2010, 16, 119-128. [CrossRef]

16. Anthony, J.C.; Yoshizawa, F.; Anthony, T.G.; Vary, T.C.; Jefferson, L.S.; Kimball, S.R. Leucine stimulates translation initiation in skeletal muscle of postabsorptive rats via a rapamycin-sensitive pathway. J. Nutr. 2000, 130, 2413-2419. [CrossRef]

17. Buse, M.G.; Reid, S.S. Leucine. A possible regulator of protein turnover in muscle. J. Clin. Investig. 1975, 56, 1250-1261. [CrossRef]

18. Katsanos, C.S.; Kobayashi, H.; Sheffield-Moore, M.; Aarsland, A.; Wolfe, R.R. A high proportion of leucine is required for optimal stimulation of the rate of muscle protein synthesis by essential amino acids in the elderly. Am. J. Physiol. Endocrinol. Metab. 2006, 291, E381-E387. [CrossRef]

19. Rieu, I.; Balage, M.; Sornet, C.; Debras, E.; Ripes, S.; Rochon-Bonhomme, C.; Pouyet, C.; Grizard, J.; Dardevet, D. Increased availability of leucine with leucine-rich whey proteins improves postprandial muscle protein synthesis in aging rats. Nutrition 2007, 23, 323-331. [CrossRef]

20. Saha, A.K.; Xu, J.; Ebony, L.; Rosangela, D.; Brandon, A.E.; Kraegen, E.W.; Ruderman, N.B. Downregulation of AMPK accompanies leucine- and glucose-induced increases in protein synthesis and insulin resistance in rat skeletal muscle. Diabetes 2010, 59, 2426-2434. [CrossRef]

21. Ventrucci, G.; Mello, M.A.R.; Gomes-Marcondes, M.C.C. Leucine-rich diet alters the eukaryotic translation initiation factors expression in skeletal muscle of tumour-bearing rats. BMC Cancer 2007, 7, 42. [CrossRef]

22. Chulay, J.D.; Haynes, J.D.; Diggs, C.L. Plasmodium falciparum: Assessment of in vitro growth by [3H]hypoxanthine incorporation. Exp. Parasitol. 1983, 55, 138-146. [CrossRef]

23. Dangoudoubiyam, S.; Zhang, Z.; Howe, D.K.; Hemphill, A. Purine salvage in the apicomplexan Sarcocystis neurona, and generation of hypoxanthine-xanthine-guanine phosphoribosyltransferase-deficient clones for positive-negative selection of transgenic parasites. Parasitology 2014, 141, 1399-1405. [CrossRef] [PubMed]

24. Morse, S.A.; Bartenstein, L. Purine metabolism in Neisseria gonorrhoeae: The requirement for hypoxanthine. Can. J. Microbiol. 1980, 26, 13-20. [CrossRef]

25. Ullman, B.; Carter, D. Hypoxanthine-guanine phosphoribosyltransferase as a therapeutic target in protozoal infections. Infect. Agents Dis. 1995, 4, 29-40.

26. Saugstad, O.D. Hypoxanthine as a measurement of hypoxia. Pediatric Res. 1975, 9, 158-161. [CrossRef]

27. Simmonds, R.J.; Coade, S.B.; Harkness, R.A.; Drury, L.; Hytten, F.E. Nucleotide, nucleoside and purine base concentrations in human placentae. Placenta 1982, 3, 29-37. [CrossRef]

28. Feet, B.A.; Yu, X.Q.; Rootwelt, T.; Oyasaeter, S.; Saugstad, O.D. Effects of hypoxemia and reoxygenation with $21 \%$ or $100 \%$ oxygen in newborn piglets: Extracellular hypoxanthine in cerebral cortex and femoral muscle. Crit. Care Med. 1997, 25, 1384-1391. [CrossRef]

29. Phillis, J.W.; O’Regan, M.H.; Walter, G.A. Effects of deoxycoformycin on adenosine, inosine, hypoxanthine, xanthine, and uric acid release from the hypoxemic rat cerebral cortex. J. Cereb. Blood Flow Metab. Off. J. Int. Soc. Cereb. Blood Flow Metab. 1988, 8, 733-741. [CrossRef] [PubMed]

30. Arruda, P.; Kemper, E.L.; Papes, F.; Leite, A. Regulation of lysine catabolism in higher plants. Trends Plant Sci. 2000, 5, 324-330. [CrossRef] 
31. Arruda, P.; Sodek, L.; Silva, W.J. Lysine-ketoglutarate reductase activity in developing maize endosperm. Plant Physiol. 1982, 69, 988-989. [CrossRef]

32. Serrano, G.C.D.M.; Figueira, T.R.E.S.; Kiyota, E.; Zanata, N.; Arruda, P. Lysine degradation through the saccharopine pathway in bacteria: LKR and SDH in bacteria and its relationship to the plant and animal enzymes. FEBS Lett. 2012, 586, 905-911. [CrossRef]

33. Higgins, A.D.; Silverstein, J.T.; Engles, J.; Wilson, M.E.; Rexroad, C.E.; Blemings, K.P. Starvation induced alterations in hepatic lysine metabolism in different families of rainbow trout (Oncorhynchus mykiss). Fish Physiol. Biochem. 2005, 31, 33-44. [CrossRef]

34. Trupin, J.S. Lysine biosynthesis in Neurospora crassa. Fed. Proc. 1963, 22, 243-250.

35. Liebmann, B.; Mühleisen, T.W.; Müller, M.; Hecht, M.; Weidner, G.; Braun, A.; Brock, M.; Brakhage, A.A. Deletion of the Aspergillus fumigatus lysine biosynthesis gene lysF encoding homoaconitase leads to attenuated virulence in a low-dose mouse infection model of invasive aspergillosis. Arch. Microbiol. 2004, 181, 378-383. [CrossRef]

36. Fu, X.; Gao, H.; Tian, F.; Gao, J.; Lou, L.; Liang, Y.; Ning, Q.; Luo, X. Mechanistic effects of amino acids and glucose in a novel glutaric aciduria type 1 cell model. PLOS ONE 2014, 9, e110181. [CrossRef]

37. Higashino, K.; Tsukada, K.; Lieberman, I. Saccharopine, a product of lysine breakdown by mammalian liver. Biochem. Biophys. Res. Commun. 1965, 20, 285-290. [CrossRef]

38. Johansson, E.; Steffens, J.J.; Lindqvist, Y.; Schneider, G. Crystal structure of saccharopine reductase from Magnaporthe grisea, an enzyme of the $\alpha$-aminoadipate pathway of lysine biosynthesis. Structure 2000, 8, 1037-1047. [CrossRef]

39. Jones, E.E.; Broquist, H.P. Saccharopine, an intermediate of the aminoadipic acid pathway of lysine biosynthesis. Ii. Studies in Saccharomyces cereviseae. J. Biol. Chem. 1965, 240, 2531.

40. Aoyagi, Y.; Sasaki, H.; Sugahara, T. Isolation and Identification of Saccharopine from Lentinus edodes. J. Agric. Chem. Soc. Jpn. 1978, 42, 1941-1942. [CrossRef]

41. Hallen, A.; Jamie, J.F.; Cooper, A.J.L. Lysine metabolism in mammalian brain: An update on the importance of recent discoveries. Amino Acids 2013, 45, 1249-1272. [CrossRef]

42. Hutzler, J.; Dancis, J. Conversion of lysine to saccharopine by human tissues. Biochim. Biophys. Acta 1968, 158, 62-69. [CrossRef]

43. Hassan, Y.; Layla, K.; Osta, M.A. The mosquito melanization response is implicated in defense against the entomopathogenic fungus Beauveria bassiana. PLoS Pathog. 2012, 8, e1003029.

44. Thierry, D.; Richard, B.; Elisabeth, G.; Pascale, C. Amino acid changes during sunflower infection by the necrotrophic fungus Botrytis cinerea. Plant Signal. Behav. 2009, 4, 859-861.

45. Ulrich, H.; Fouad, O.; Franz-Josef, M.; Hermann, B. The bacterium Paenibacillus validus stimulates growth of the arbuscular mycorrhizal fungus Glomus intraradices up to the formation of fertile spores. FEMS Microbiol. Lett. 2006, 254, 258-267.

46. Mchunu, N.P.; Permaul, K.; Alam, M.; Singh, S. Carbon utilization profile of a thermophilic fungus, Thermomyces lanuginosus using phenotypic microarray. Adv. Biosci. Biotechnol. 2013, 4, 24-32. [CrossRef]

47. Genghof, D.S.; Vandamme, O. Biosynthesis of ergothioneine and hercynine by mycobacteria. J. Bacteriol. 1964, 87, 852-862.

48. Dirk, G.; Stephanie, H.; Stefan, G.; Andreas, G.; Andreas, L.; Reinhard, B.; Norma, J.; Andrea, R.; Edgar, S.M. Discovery of the ergothioneine transporter. Proc. Natl. Acad. Sci. USA 2005, 102, 5256-5261.

49. Melville, D.B.; Horner, W.H.; Otken, C.C.; Ludwig, M.L. Studies on the origin of ergothioneine in animals. J. Biol. Chem. 1955, 213, 61-68.

50. Cumming, B.M.; Chinta, K.C.; Reddy, V.P.; Steyn, A.J. Role of ergothioneine in microbial physiology and pathogenesis. Antioxid. Redox Signal. 2018, 28, 431-444. [CrossRef]

51. Askari, A.; Melville, D.B. The reaction sequence in ergothioneine biosynthesis: Hercynine as an intermediate. J. Biol. Chem. 1962, 237, 1615-1618.

52. Seebeck, F.P. In vitro reconstitution of Mycobacterial ergothioneine biosynthesis. J. Am. Chem. Soc. 2010, 132, 6632-6633. [CrossRef] [PubMed]

53. Servillo, L.; Castaldo, D.; Casale, R.; D’Onofrio, N.; Giovane, A.; Cautela, D.; Balestrieri, M.L. An uncommon redox behavior sheds light on the cellular antioxidant properties of ergothioneine. Free Radic. Biol. Med. 2015, 79, 228-236. [CrossRef] [PubMed] 
54. Zhu, B.Z.; Mao, L.; Frei, B. Ergothioneine prevents copper-induced oxidative damage to DNA and protein by forming a redox-inactive ergothioneine-copper complex. Chem. Res. Toxicol. 2011, 24, 30. [CrossRef] [PubMed]

55. Akanmu, D.; Cecchini, R.; Aruoma, O.I.; Halliwell, B. The antioxidant action of ergothioneine. Arch. Biochem. Biophys. 1991, 288, 10-16. [CrossRef]

56. Franzoni, F.; Colognato, R.; Galetta, F.; Laurenza, I.; Barsotti, M.; Stefano, R.D.; Bocchetti, R.; Regoli, F.; Carpi, A.; Balbarini, A.; et al. An in vitro study on the free radical scavenging capacity of ergothioneine: Comparison with reduced glutathione, uric acid and trolox. Biomed. Pharmacother. 2006, 60, $453-457$. [CrossRef]

57. Chaudière, J.; Ferrari-Iliou, R. Intracellular antioxidants: From chemical to biochemical mechanisms. Food Chem. Toxicol. 1999, 37, 949-962. [CrossRef]

58. Bello, M.H.; Viviana, B.P.; Dexter, M.; Lynn, E. The Neurospora crassa mutant Nc $\Delta$ Egt-1 identifies an ergothioneine biosynthetic gene and demonstrates that ergothioneine enhances conidial survival and protects against peroxide toxicity during conidial germination. Fungal Genet. Biol. 2012, 49, 160-172. [CrossRef]

(C) 2019 by the authors. Licensee MDPI, Basel, Switzerland. This article is an open access article distributed under the terms and conditions of the Creative Commons Attribution (CC BY) license (http://creativecommons.org/licenses/by/4.0/). 